This item was submitted to Loughborough's Research Repository by the author.

Items in Figshare are protected by copyright, with all rights reserved, unless otherwise indicated.

\title{
Crowd simulation: A video observation and agent-based modelling approach
}

PLEASE CITE THE PUBLISHED VERSION

http://dx.doi.org/10.1504/IJDH.2016.10000735

\section{PUBLISHER}

(C) Inderscience

\section{VERSION}

AM (Accepted Manuscript)

\section{PUBLISHER STATEMENT}

This work is made available according to the conditions of the Creative Commons Attribution-NonCommercialNoDerivatives 4.0 International (CC BY-NC-ND 4.0) licence. Full details of this licence are available at: https://creativecommons.org/licenses/by-nc-nd/4.0/

\section{LICENCE}

CC BY-NC-ND 4.0

\section{REPOSITORY RECORD}

Mohamaddan, Shahrol, and Keith Case. 2019. "Crowd Simulation: A Video Observation and Agent-based Modelling Approach”. figshare. https://hdl.handle.net/2134/23036. 


\title{
Crowd Simulation: A Video Observation and Agent-based Modelling Approach
}

\section{Shahrol Mohamaddan}

Department of Mechanical and Manufacturing, Faculty of Engineering, Universiti Malaysia Sarawak (UNIMAS), 94300 Kota Samarahan, Sarawak, Malaysia mshahrol@feng.unimas.my

\section{Keith Case}

Mechanical and Manufacturing Engineering, Loughborough University, Leicestershire, LE11 3TU, United Kingdom

K.Case@lboro.ac.uk

\begin{abstract}
Human movement in a crowd can be considered as complex and unpredictable, and accordingly large scale video observation studies based on a conceptual behaviour framework were used to characterise individual movements and behaviours. The conceptual behaviours were Free Movement (Moving Through and Move-Stop-Move), Same Direction Movement (Queuing and Competitive) and Opposite Direction Movement (Avoiding and Passing Through). Movement in crowds was modelled and simulated using an agent-based method using the gaming software Dark BASIC Professional. The agents (individuals) were given parameters of personal objective, visual perception, speed of movement, personal space and avoidance angle or distance within different crowd densities. Two case studies including a multi-mode transportation system layout and a bottleneck / non-bottleneck evacuation are presented.
\end{abstract}

Keywords: Agent-based modelling, crowd simulation, observational study

\section{Introduction}

A crowd can be defined as a situation where humans flock together like other aggregations of animals (e.g. herds of cattle and schools of fish) or material (e.g. fluid and particle flow) (Henein et al. 2007). At times of stress or panic such as in an emergency evacuation individual decisiontaking may be abandoned and the crowd reacts as a single organism. A crowd can also be defined as a large group of individuals in the same physical environment and sharing a common goal (Musse et al. 1997). An 
example here would be large numbers of people trying to enter or leave a sports stadium. Finally, there are many crowded places including for example train stations and shopping malls where the individuals each have their own objective but their behaviour is modified by the presence of others. It is this latter situation which is the focus for the work reported here. The work has its origins in the AUNT-SUE (Accessibility and User Needs - Sustainable Urban Environments) research programme (Marshall et al., 2009). This was a large Engineering and Physical Sciences Research Council (EPSRC) funded programme concerned with inclusive design in urban transport environments. i.e. there was a focus on older people and people with disabilities, which has been carried on into this work.

\section{Literature Survey}

Many researchers have published work related to human movement and behaviour in crowds where the focus is more on the emergency evacuation situation. The emphasis is to avoid the occurrence of dangerous crowd phenomena (e.g. arising from fire hazards, accidents, earthquakes etc.), to reduce the numbers of people injured or killed should it occur and to improve the environment for the crowd in terms of the quality of the facilities, signage and communications. The crowd dynamics research by Still (2000) is a typical example of this area.

Crowd modelling and simulation research involves developing a model to represent the crowd or individuals in a crowd. Based on the developed model, the crowd or individuals in the crowd are simulated in a virtual environment. In general, crowd modelling and simulation is based on mathematical equations or sets or rules representing different situations or conditions of the crowd or individuals in the crowd. However, most of the models are a simplification since the movement of the crowd or individuals is considered to be very diverse and complex.

There are several reviews of crowd modelling and simulation research. For example, Zheng et al. (2009) reviewed seven types of crowd models for evacuation from buildings, classifying them into selected features (the approaches, individual/groups, scale, space and time, situations and typical phenomena applied to the model) in order to differentiate the models. Papadimitriou et al., (2009) reviewed several crowd models related to pedestrian behaviour in urban areas. The crowd models were reviewed based on two aspects; firstly pedestrian movement and route choice and secondly pedestrian crossing behaviour. Olenick and Carpenter (2003) reviewed computer models related to fire and smoke as an update to previous work conducted by Friedman (1992). Although the review was 
not specifically related to the crowd issue, most of the selected models were applied in crowd modelling and simulation research. Four modelling methods (Cellular Automata, Social Force, Lattice Gas and Agent-based models) are briefly discussed here.

Cellular Automata (CA) are discrete dynamic systems that model complex behaviour based on simple, local rules animating cells in a lattice. CA models have been used in various complex systems including physics, biology and traffic modelling. The cell objects in the CA are located in a regular grid where each cell has its own state. The number of state possibilities is typically finite and the simplest example has the two possibilities of 1 and 0 (can be referred as "on" and "off" or "alive" and "dead"). Each cell has a neighbourhood that can be defined in a number of ways. The initial state is selected by assigning a state for each cell. A new state of each cell is created according to some fixed rule such as a mathematical function. The updating process in CA exhibits similar behaviour to biological reproduction and evolution. Schadschneider (2002) modelled each particle (human) walking directions in a $3 \times 3$ matrix of preferences that contain the probabilities of the movement. The central element describes the probability for the particle not to move at all. The remaining 8 correspond to a move to the neighbouring cells. The matrix of preferences contains information about the preferred walking direction and speed. The walking direction and speed can be different from cell to cell depending on the geometry and aim of the pedestrians. In crowd research, CA have been applied in various problem domains including the study of exit dynamics of occupant evacuation (Daoliang et al., 2006) and the exit dynamics of pedestrians in a room (Perez et al., 2002). CAs have also been applied to study the effect of obstacles by simulating the evacuation process from a room with and without obstacles and to simulate bi-directional pedestrian movement (Fang et al., 2003). According to Teknomo (2002), a CA model is simple to develop and fast in updating the data. However, the heuristic approach in updating the rules is undesirable since a CA model does not reflect the real behaviour of humans. The inherent grid of cells of a CA model can also make the behaviour of the human seem visually rough and gives the impression that the human is jumping from one cell to another.

A lattice gas model is a series of Cellular Automata models used to simulate fluid flows. As in cellular automaton, the lattice gas model comprises a lattice where the sites on the lattice (the intersection points of the lattice) can take a certain number of different states. In this model, the various states refer to the particles with certain velocities. The lattice gas model has been applied in various problems such as random pedestrian 
flow in channels through bottlenecks (Tajima et al., 2001), simulating crowd flow in a hall (Tajima at al., 2001), simulating pedestrian flow in a T-shaped channel (Tajima and Nagatani, 2002), simulating counter flow in a channel (Nagai at al., 2005) and simulating the evacuation process from a classroom (Helbing at al., 2003). Lattice gas models have also been combined with other approaches to investigate the evacuation process. For example, a multi-grid model (lattice gas concept) with the force concept of a social force model to simulate interaction forces among the pedestrian (Zhang at al., 2008).

The social force model was proposed by Helbing and Molnar (1995) as a pedestrian motion model and Mehran at al. (2009) produced a generalised model. In this model, due to individualistic goals or environmental constraints, each human has a mass and changes velocity as a result of a force. The force consists of a personal desire force and an interaction force. The personal desire force refers to the desired direction and velocity that humans have when seeking certain goals and destinations in the environment. However, the crowd limits individual movement and the actual velocity differs from the desired velocity. The interaction force consists of the repulsive and attraction forces between humans based on a psychological tendency to keep a social distance (personal space) and an environment force to avoid hitting walls, buildings and other obstacles. The social force model has also been applied in many areas of research. Seyfried at al. (2006) applied a social force model to simulate pedestrian dynamics and qualitatively analyse the influence of various approaches for the interaction between the pedestrians on the resulting velocity-density relationship. The social force model has also been applied in other crowd research such as crowd monitoring (Mehran at al., 2009) to observe public areas using image processing analysis.

Agent-based modelling (ABM) is a computational simulation method based on computer-driven agents. According to Schieritz and Milling (2003) there is no universally accepted definition of ABM especially on the kind of properties an object has if it deserves to be called an 'agent' including pro- and re-activeness, spatial awareness, ability to learn, social ability, intellect, etc. In crowd research, the agents are the human entities modelled in the virtual environment. The agents are represented by an identifiable unit of computer program code which is autonomous and goaldirected. Autonomous refers to the capability of an agent to perform independent action while goal-directed refers to autonomous action that is directed towards the achievement of defined tasks. In general, the agents are designed with different characteristics at the individual level. When the simulation is performed, the global behaviour emerges as a result of 
many (tens, hundreds, thousands, etc.) individuals, each following its own behaviour rules and interacting with each other and with their environment. $\mathrm{ABM}$ is associated with a programming technique called object-oriented programming. ABM has been applied to various problem domains. For example Augustijn-Beckers at al. (2010) applied ABM to simulate the effects of pre-evacuation behaviour and exit choice strategies. The agents were divided into three categories (or behaviours) known as Officers, Leavers and Followers. The officers were modelled as trained emergency personal with the main behaviour of clearing the building by moving to leavers or followers who have not yet started the evacuation process to prompt them to evacuate. Leavers represent people that can evacuate on their own with the main behaviour of the ability to make an exit choice. Lastly, the followers represent people that do not evacuate independently but have to evacuate with either a "leaver" or "officer". Pan et al. (2006) applied ABM to simulate the social behaviour of humans during emergency situations. The simulation model was categorised into three levels that consisted of different rules; the individual, the interactions among individuals and the group and the environment. The three levels developed in the research were the examination of crowd behaviour from the perspectives of human decision-making and social interaction. The simulation was able to simulate social behaviours such as competitive, queuing and herding behaviours. Schelhorn at al. (1999) applied ABM to simulate and predict pedestrian behaviour in urban centres. The agents were developed based on two broad characteristics; socio-economic and behavioural. The socio-economic characteristic refers to the income and gender of the agent. Both characteristics were used to create the activity for the agent such as the sequence of locations which the agents intend to visit in the town centre. On the other hand, the behavioural characteristic refers to detailed behaviour of the agents including the speed, visual range and agents' focus to follow the scheduled activity.

This paper discusses crowd modelling and simulation based on sets of rules or an agent-based approach using gaming software called DarkBASIC Professional (DBPro). The focus is on the movement and behaviour of individual humans in normal crowded situations (where no panic situation is involved) and the interactions between individuals is in localized areas. An emphasis is placed on gaining knowledge of some of the characteristics of human movement and behaviour in the real world so that these can be modelled in the virtual environment. 


\section{Video Observation Studies}

The video observational method is widely applied in ergonomics and image processing research. In ergonomics research, video observational methods have been preferred over self-reporting because it offers better validity and reliability (Pehkonen et al. 2009). The method has been widely applied to observe whole body movement such as assessing the load in low back, shoulders and lower extremities. According to Dempsey et al. (2005) and Pascual et al. (2008) although there is still no universal method to observe all kinds of jobs, a wide variation of observational methods have been applied in ergonomics research.

One of the prominent characteristics of systematic observation is the use of a predefined list of behavioural codes that have been developed for particular observational studies. The coding schemes were the instruments of measurement for observational research where the behaviours were specified based on the research objective (Noldus et al. 2000). Besides developing predefined lists of behavioural codes that reflected specific needs, there are many examples of behavioural codes developed to assist observational studies such as Rapid Upper Limb Assessment (RULA) (McAtamney et al. 1993), Rapid Entire Body Assessment (REBA) (Hignett et al. 2000), Ovako Working Posture Analysis System (OWAS) and Quick Exposure Check (QEC) (David et al. 2008).

The video observational method provides an opportunity to observe some unpredictable or natural movement and behaviour of humans, although the issues of privacy and security require serious consideration. In this research, the video observational method was applied as an input to analysing human movement and behaviour in crowded areas from the real world. Figure 1 shows an overview of the steps taken to understand and analyse human movement and behaviour from the recorded video. The video observational method starts with recording the crowd at the selected location and time. The recorded video was viewed once to get an overview of the crowd location to reflect on the research objective. 

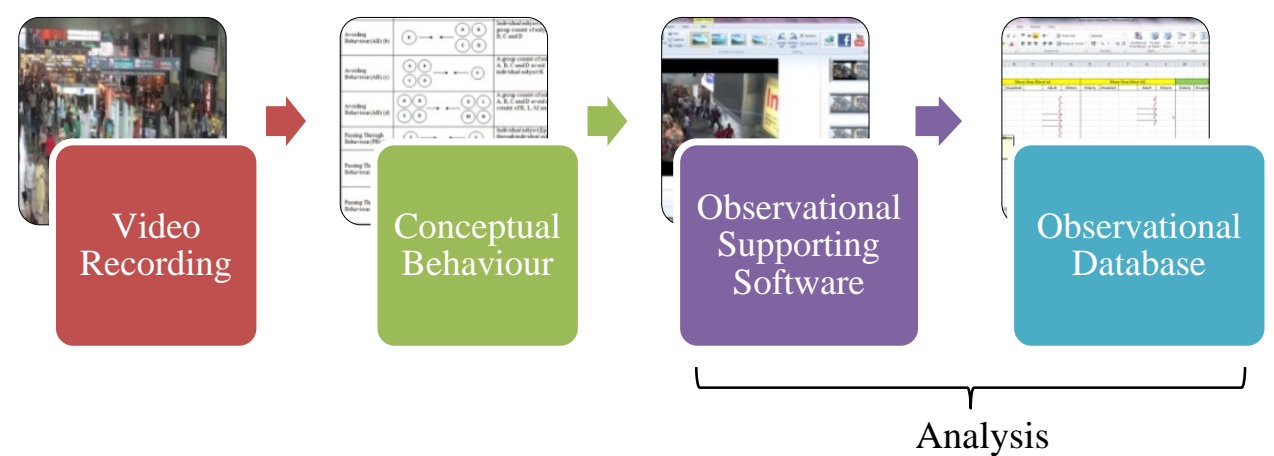

Figure 1 The observational flow

Subsequently, conceptual behaviours were developed to assist the observation process. The conceptual behaviours are a set of behaviour rules or codes that have been developed based on the major human movements of free, opposite and same direction. The conceptual behaviour set becomes the reference document to be referred to in order to avoid any confusion or exclusion of any humans during the analysis process. The analysis process is the last step taken in the process where the observational supporting software and observational database is applied for that purpose. Video recordings were made on a weekday and on a weekend at three different times of the day. A total of 18,946 individuals were observed (and their behavior subsequently analysed) - see table 1 .

Table 1 Video observational subjects

\begin{tabular}{|c|c|c|}
\hline \multirow{2}{*}{ Recording session } & \multicolumn{2}{|c|}{ Number of subjects } \\
\cline { 2 - 3 } & Weekend & Weekday \\
\hline $\begin{array}{c}\text { Morning (7:30 am - 8:30 } \\
\text { am) }\end{array}$ & 1,152 & 3,020 \\
\hline $\begin{array}{c}\text { Afternoon (12:00 pm - } \\
\text { 1:00 pm) }\end{array}$ & 3,402 & 4,182 \\
\hline $\begin{array}{c}\text { Evening (5:00 pm - 6:00 } \\
\text { pm) }\end{array}$ & 3,254 & 3,936 \\
\hline Total subjects & 7,808 & 11,138 \\
\hline Grand Total Subjects & \multicolumn{2}{|c|}{$\mathbf{1 8 , 9 4 6}$} \\
\hline
\end{tabular}


In this research, part of a transportation area called Stesen Sentral Kuala Lumpur as shown in Figure 2 was selected as the video recording location. Stesen Sentral Kuala Lumpur is the largest transportation hub or transfer station in Malaysia integrating all major transportation networks including trains, buses and taxis services from Kuala Lumpur to other areas in Malaysia.

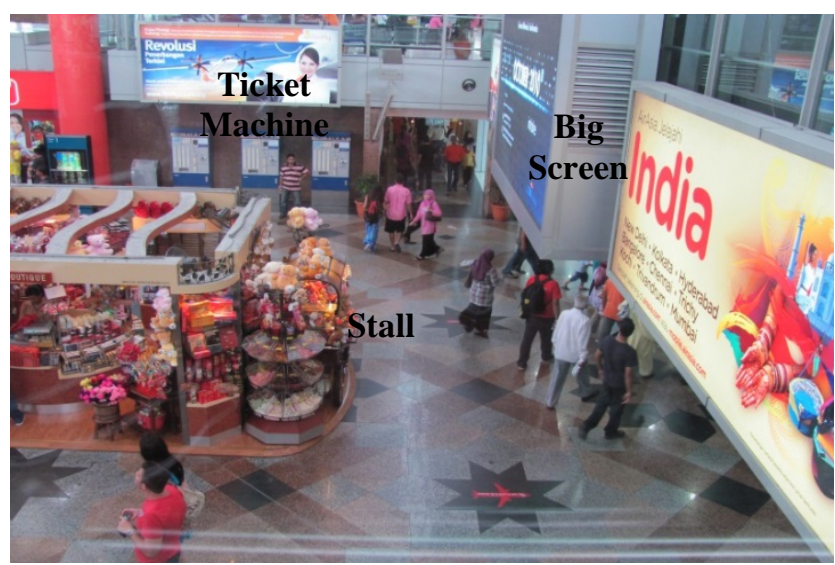

Figure 2 Stesen Sentral, Kuala Lumpur

The transfer station has special characteristics to be considered for crowd modelling and simulation such as differences in activities, time pressure and movement patterns (Daamen et al. 2002). The activities refer to different types of activities that might arise at the transfer station such as waiting for other people, buying tickets, shopping and others. Time pressure refers to conditions where there is a possibility to observe people either hurrying to catch the public transport (such as train or bus) and in more relaxed situations. It is in the nature of transfer station that movement patterns of the crowd are based on arrivals/departures determined by the public transportation timetable.

\section{Conceptual Behaviours and Analysis}

Conceptual behaviours are a set of behaviours rules or codes that was developed to assist the observational analysis from the recorded video. The conceptual behaviours were developed based on observing the physical movement of individual humans in crowded areas (without any contact or direct communication) and the understanding of human movement and behaviour.

The process starts with defining the focus subject for the research which is the individual person in a crowd. Each individual person in a 
crowd will be observed and the focus is on the movements that are performed known as free, same and opposite direction movement.

Free movement is considered as the basic human movement from one point to another point of interest. In free movement, a person is considered to have enough space to move without facing any obstacle or constraint during the movement. There are two types of behaviours that can occur during free movement; Moving Through and Move-Stop-Move behaviour. Moving Through is behaviour where the person just moves through the observation area without any specific concern. The only difference that can be observed between people is the speed of movement. Move-StopMove is the behaviour where people have to stop a few seconds or a few minutes to perform some other related task during movement such as buying a ticket, using a mobile phone, waiting for someone or looking for information.

Same direction movement consists of Queuing and Competitive behaviour. The Queuing and Competitive behaviours can easily be observed when people enter or exit a door. Queuing behavior is the manifestation of self-organizing where individual people create a line to enter or exit the door. On the other hand, in competitive behaviour people have less consideration for each other and each individual tries to enter or exit the door as quickly as possible.

Opposite direction refers to the condition where a person meets another person travelling in the opposite direction, for example when moving along a pathway. Upon meeting each other during the movement there are two possible behaviours that can occur; Avoiding and Passing Through. Avoiding refers to the behaviour where a person gives way to other people or performs an avoidance route. On the other hand, Passing Through refers to the behaviour where people do not consider others or their surroundings during the movement. The major concern is to reach the targeted area within the planned route.

In conceptual behaviours, human movement was considered as a scenario to acquire the human behaviour or behaviour pattern. The idea of having behaviour patterns from movement scenarios was based on scenario building that has been developed in product design. Scenario building is a tool for exploration, prototyping and communication at an early stage of the product design process.

The scenario is defined as a set of users, a context and a set of tasks that users perform or want to perform (Fulton Suri et al. 2000). Thus, the conceptual behaviour is a foundation for what is going to be observed and analysed so that better insight into human behaviour can be achieved 
whilst avoiding any confusion during the observation process due to the large volume of movement and behaviour within the recorded video.

Each frame of the six hours of video was viewed for 30 seconds using the observational supporting software. Thirty seconds of video frame was considered as a manageable time for analysis whilst avoiding any confusion. Each individual in the 30 seconds of video frame was observed manually one by one to classify the types of behaviour performed by the individual and an observational database constructed. The observed behaviours are summarised in table 2 .

Table 2 Human behaviour based on movement of subjects

\begin{tabular}{|l|c|c|c|c|c|}
\hline \multicolumn{1}{|c|}{ Behaviour } & $\begin{array}{c}\text { Older } \\
\text { People }\end{array}$ & $\begin{array}{c}\text { People } \\
\text { with } \\
\text { Disabi } \\
\text { lities }\end{array}$ & Adults & Others & TOTAL \\
\hline Free Direction Movement & 268 & 15 & 15,192 & 388 & $\mathbf{1 5 , 8 6 3}$ \\
\hline Moving Through & 71 & 1 & 1,770 & 87 & $\mathbf{1 , 9 2 9}$ \\
\hline Move-Stop-Move & 1 & 0 & 162 & 1 & $\mathbf{1 6 4}$ \\
\hline Same Direction Movement & 2 & 0 & 98 & 1 & $\mathbf{1 0 1}$ \\
\hline Queuing behaviour & 13 & 0 & 588 & 8 & $\mathbf{6 0 9}$ \\
\hline Competitive behaviour & 14 & 0 & 248 & 18 & $\mathbf{2 8 0}$ \\
\hline Opposite Direction Movement & $\mathbf{3 6 9}$ & $\mathbf{1 6}$ & $\mathbf{1 8 , 0 5 8}$ & $\mathbf{5 0 3}$ & $\mathbf{1 8 , 9 4 6}$ \\
\hline Avoiding Behaviour & & & & \\
\hline Passing Through Behaviour & TOTAL & & & \\
\hline
\end{tabular}

\section{Agent-Based Modelling}

The agent-based modelling is a bottom up computational model that simulates individuals as 'agents' within the virtual environment. In this research the individual agent was developed using gaming software, DarkBASIC Professional (DBPro) software. Figure 3 shows the adult 
entity in 3D design. There are six different parameters established for each entity.

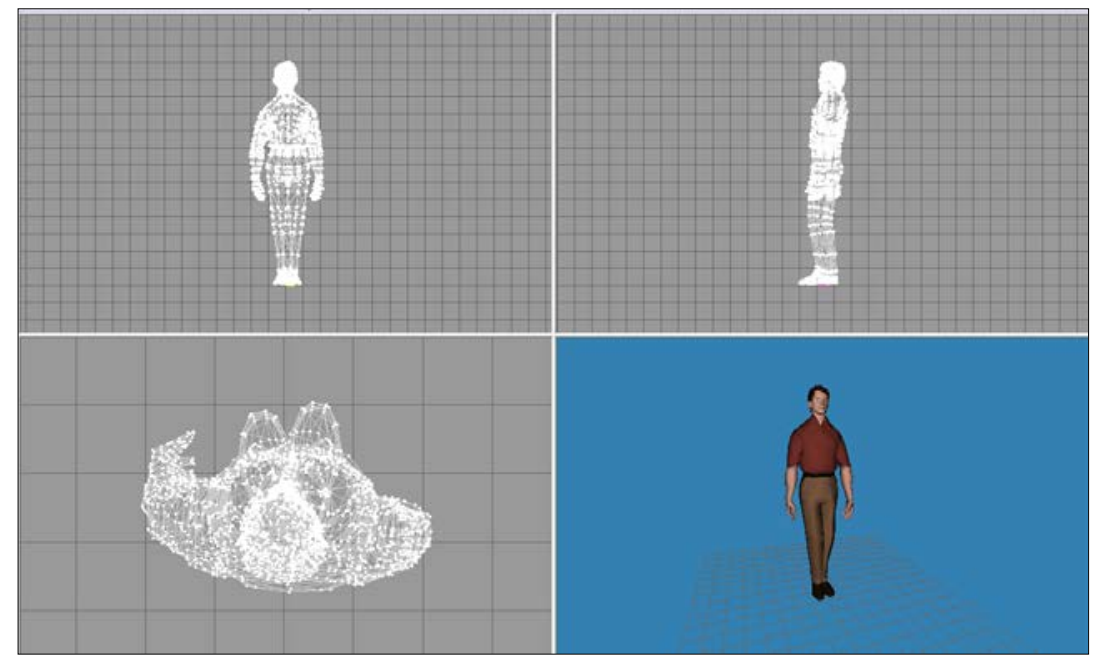

Figure 3 Adult entity in 3D design (Wai Loon, 2011)

The parameters were the factors affecting human movement and behaviour observed during the video observation study. The parameters play important roles in developing different types of simulation that utilize different movement scenarios in the real world. The parameters are personal objective, visual perception, speed of movement, personal space, crowd density and avoidance angle or distance.

\subsection{Personal Objective}

Personal objective refers to the goal that a person has during movement and can be observed from physical appearance. For example, people move around with luggage or backpacks, looking for information, buying tickets at ticket machines and other activities. The personal objective is the main reason that determines human decision making and behaviour during movement in a crowd.

In the virtual environment, the personal objective is represented by the command Position Object and AI Entity Go to Position. The Position Object command defines the initial position for each entity in the virtual environment before the entities perform any movement. The entities move towards the selected location using the AI Entity Go to Position command. The personal objective parameter in the virtual environment is shown as the red path in Figure 4. In this research, the personal objective parameters 
(the Position Object and AI Entity Go to Position command) are applied to develop the selected movements as shown in the case study simulations.

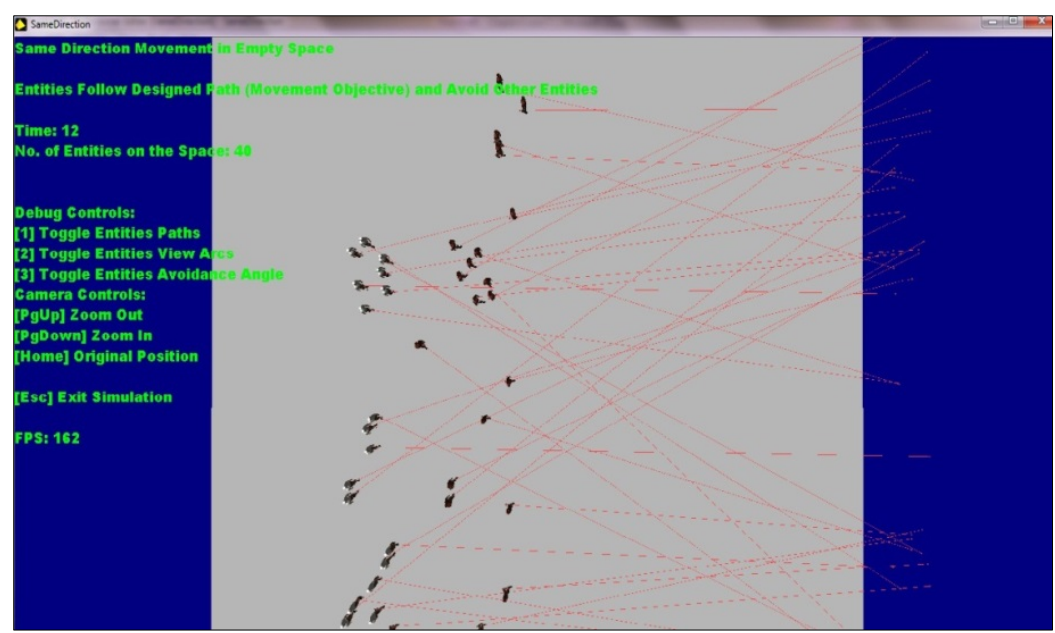

Figure 4 Personal Objective parameter in the virtual environment

\subsection{Visual Perception}

Visual perception refers to the ability of humans to process and interpret information from the surrounding environment through their visual system. According to Ondrej et al. (2010), humans rely on visual perception during movement for collision-free navigation especially in crowded areas with numerous obstacles. 


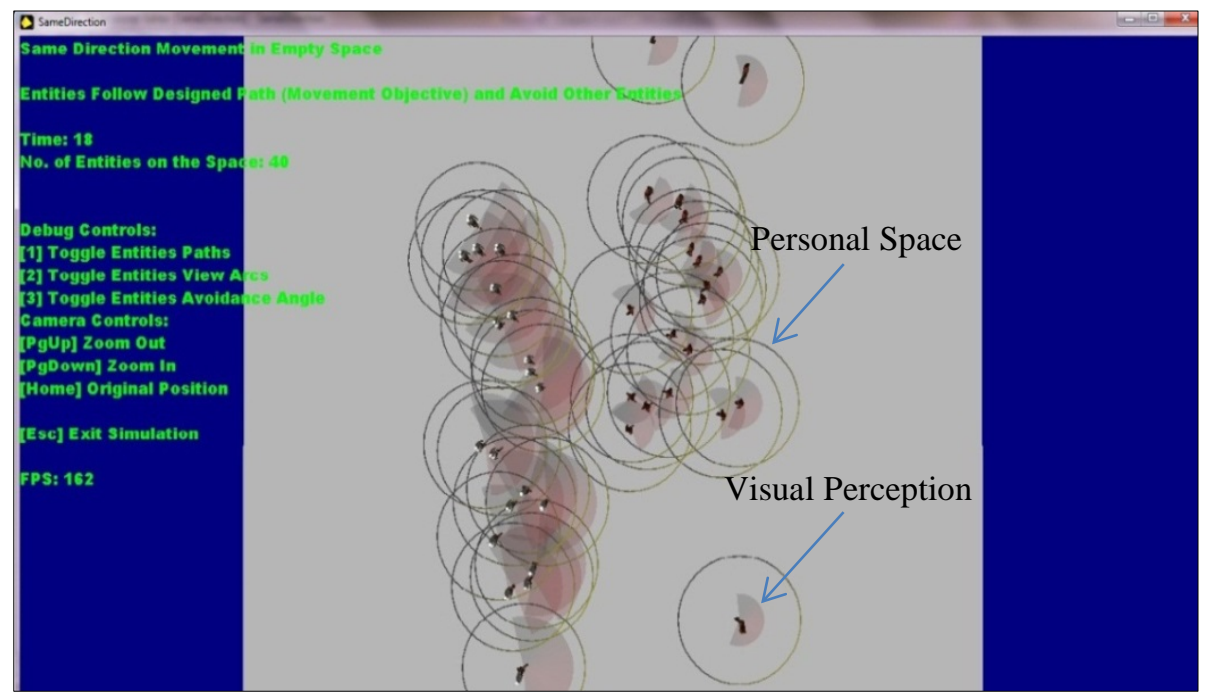

Figure 5 Personal Space and Visual Perception parameter in the virtual environment

The Visual Perception parameter in the virtual environment is represented by the view arc and the view range as shown in Figure 5. The view arc is the angle at which the entities can see their surroundings. In DarkBASIC the value of the view arc is in the range 1 to 360 degrees and is defined by the AI Set Entity View Arc command. The view range is the distance the entities can see from the current location and is defined by the AI Set Entity View Range command. The entities in the virtual environment are able to see anything that appears within the view range and the view angle.

\subsection{Speed of Movement}

Speed of movement refers to the walking speed of humans, classified into slow, average, fast and extra fast. According to Syed Shazali (2010), a slow speed can be observed when humans have limited physical ability, such as older people walking with a cane. Average speed of movement can be observed when people are walking alone without obstruction or distraction on a level walking pathway. Jogging slowly or overtaking others from a tailgating state is an example of fast speed of movement and the extra fast speed of movement can be observed when people fast jog or run.

The Speed of Movement in the virtual environment is represented by the command AI Set Entity Speed. The command sets the movement speed of the entity in DarkBASIC units per second (unit pixels). Based on the 
Wai Loon (2011), the 1 unit pixel (DarkBASIC unit) is equal to $5 \mathrm{~cm}$ in the real world. The value was used in the source code to determine the speed of movement of the virtual humans along with the work from Shirish (2001) and Teknomo (2002).

The adult entity is set to be 24 unit pixels per second or $1.2 \mathrm{~m} / \mathrm{s}$ speed of movement. The view arc is set between 160 to 210 degrees with the view range of 40 pixels or $2 \mathrm{~m}$. The adult entity is displayed blue. The older entity is set to have 16 unit pixels per second or $0.8 \mathrm{~m} / \mathrm{s}$ speed of movement. The view arc is set between 140 to 190 degrees with the view range of 20 unit pixels or $1 \mathrm{~m}$ and is displayed red.

\subsection{Personal Space}

Personal space refers to the invisible surroundings or territory around people. Personal space is considered as a social condition that affects communication or contact between humans. Personal space is divided into four categories known as the Intimate, Personal, Social Consultative and Public Zones based on the distance between humans within public spaces (Syed Shazali, 2010).

The Personal Space in the virtual environment is represented by the $A I$ Set Radius command. The command sets the global radius that is used by all entities in the virtual environment. The command controls how much space is allowed between obstacles and the waypoints that define movement around the entities. Since the entities use the waypoints to navigate around the virtual environment, the entities will attempt to maintain at least the AI Set Radius distance from all obstacles. The Personal Space parameter was illustrated previously in Figure 5.

\subsection{Crowd Density}

Crowd density refers to the number of people per square meter for stationary or moving crowds. Human speed of movement is decreased when the crowd density is increased especially during peak periods.

The crowd density was designed at three levels namely low, medium and high as shown in Table 3. 
Table 3 Definition of crowd densities

\begin{tabular}{l|l}
\hline Type of Crowd & \multicolumn{1}{c}{ Definition of the Crowd } \\
\hline Low Density & 30 entities within a $30 \mathrm{~m} \times 30 \mathrm{~m}$ area \\
\hline $\begin{array}{l}\text { Medium } \\
\text { Density }\end{array}$ & 60 entities within a $30 \mathrm{~m} \times 30 \mathrm{~m}$ area \\
\hline High Density & 90 entities within a $30 \mathrm{~m} \times 30 \mathrm{~m}$ area \\
\hline
\end{tabular}

\subsection{Avoidance Angle or Distance}

Avoidance is an action performed by people so as not to collide with other people or obstacles. Avoidance occurs when people avoid other people travelling in the opposite direction or when overtaking others travelling in the same direction. Avoidance Angle or Distance refers to the situation where the entities in the virtual environment avoid each other during the movement.

In this research, the Avoidance Angle or Distance is based on the DarkAI effect from the movement as shown in Figure 6. The Avoidance Angle or Distance is represented by the green areas in the figure. The Avoidance Angle or Distance is also affected by other parameters such as Visual Perception and Personal Space. When the floor area becomes more crowded (increased number of entities), the avoidance behaviour is observed to occur many times. 


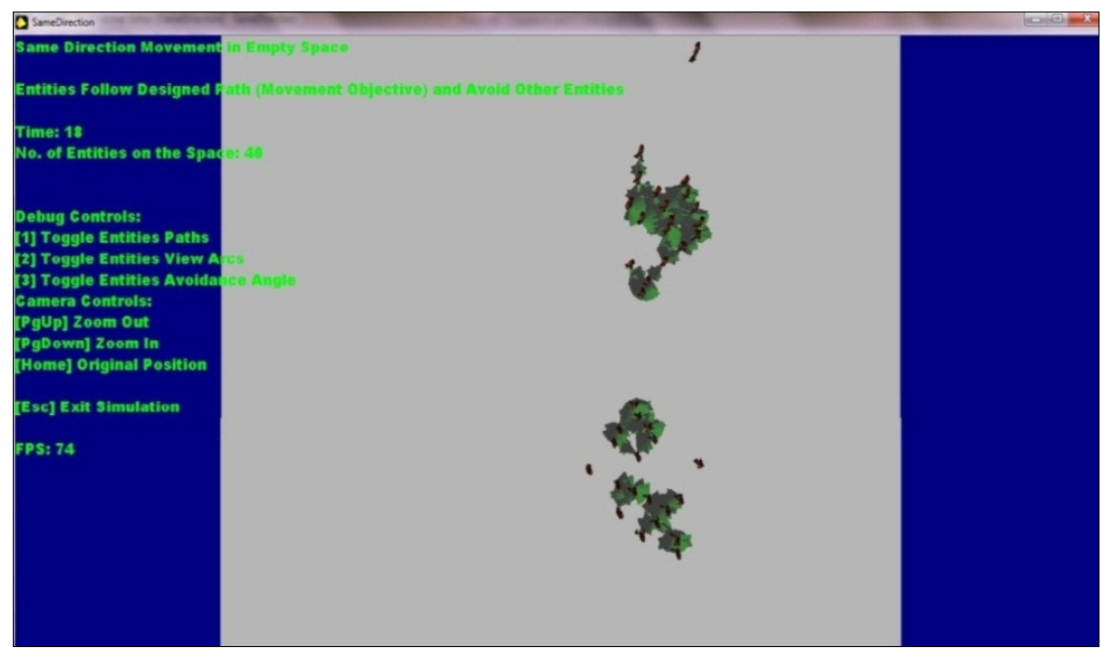

Figure 6 Avoidance Angle or Distance parameter in the virtual environment

\section{Case Study Simulation}

\subsection{Comparing Different Design Layouts}

Two different layouts were developed to represent the multi-mode transportation system as shown in Figure 7. The layout consisted of a ticket machine, waiting area, information centre, big screen, showcase area and stalls. The layouts were developed based on the observation area at Stesen Sentral, Kuala Lumpur (as shown in Figure 2) with some modification on the arrangement of the stall and showcase area.

In Layout 1 the arrangement of the stalls was in two rows where the middle of the stalls created a limited space for people to move around while the showcase area iwas arranged in two rows. On the other hand, in Layout 2 the stalls were arranged in four locations while the showcase area was arranged as a single row. The simulation was conducted to compare the effect of different layouts on the movement of people in the multi-mode transportation system. 


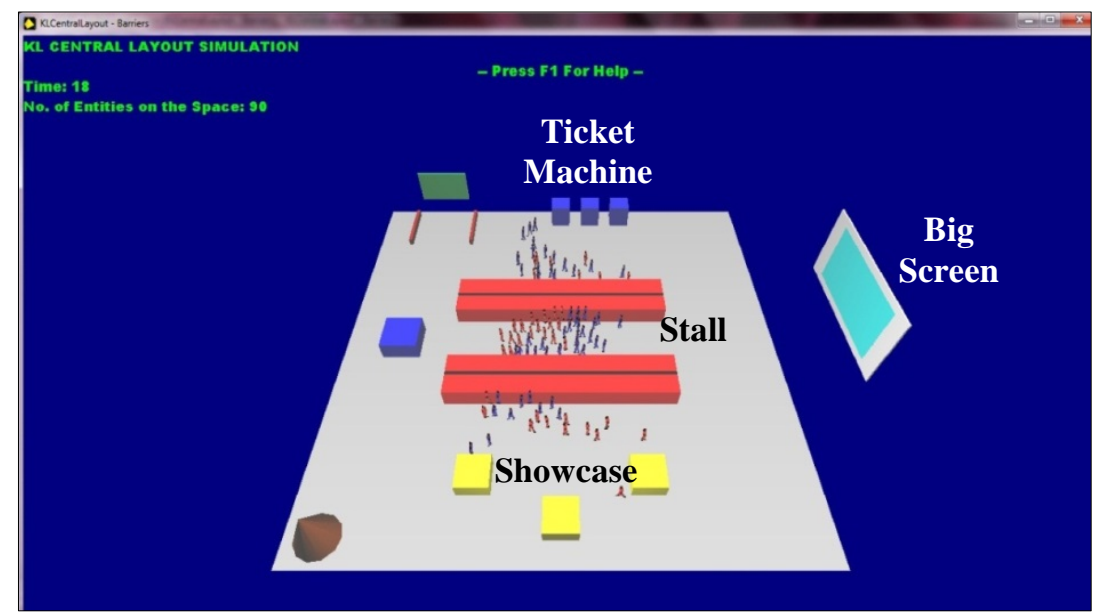

(a) Layout 1

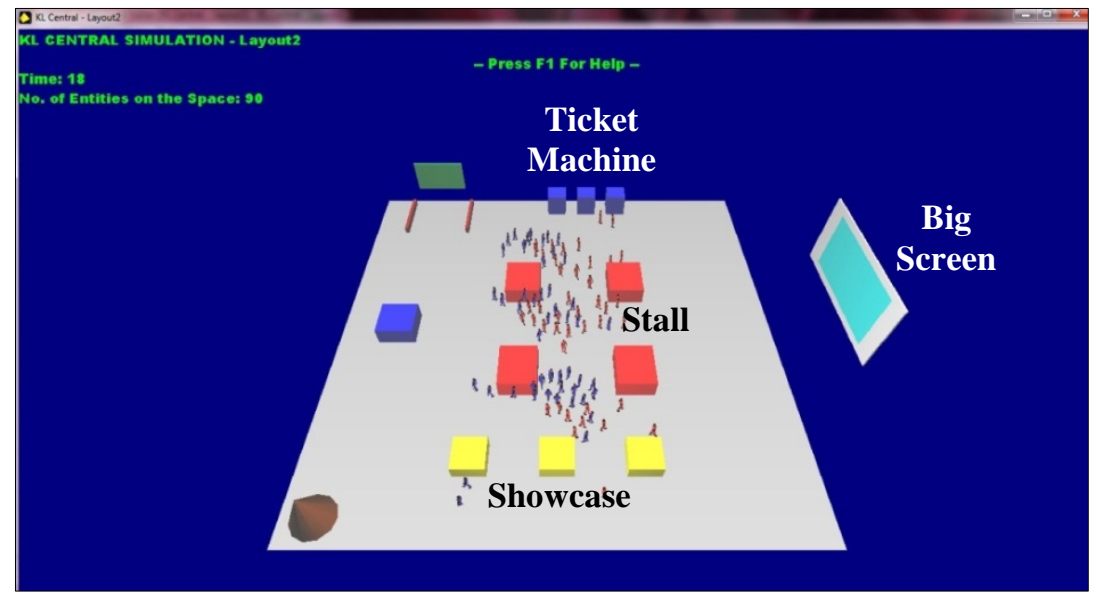

(b) Layout 2

Figure 7 Different layouts for a multi-mode transportation system simulation

\subsection{Evacuation at Bottleneck and Non-bottleneck Layouts}

The simulation for evacuation at bottleneck and non-bottleneck layouts is shown in Figure 8. The simulation was conducted to understand the effects of bottleneck and non-bottleneck layouts on evacuation space. Ninety adult entities were randomly allocated to initial positions within the two layouts. All entities were required to move towards the exit door marked by the red circle in the figure. During the simulation, the evacuation time for each entity was recorded and the results of the evacuation time from the bottleneck and non-bottleneck layouts were compared. 


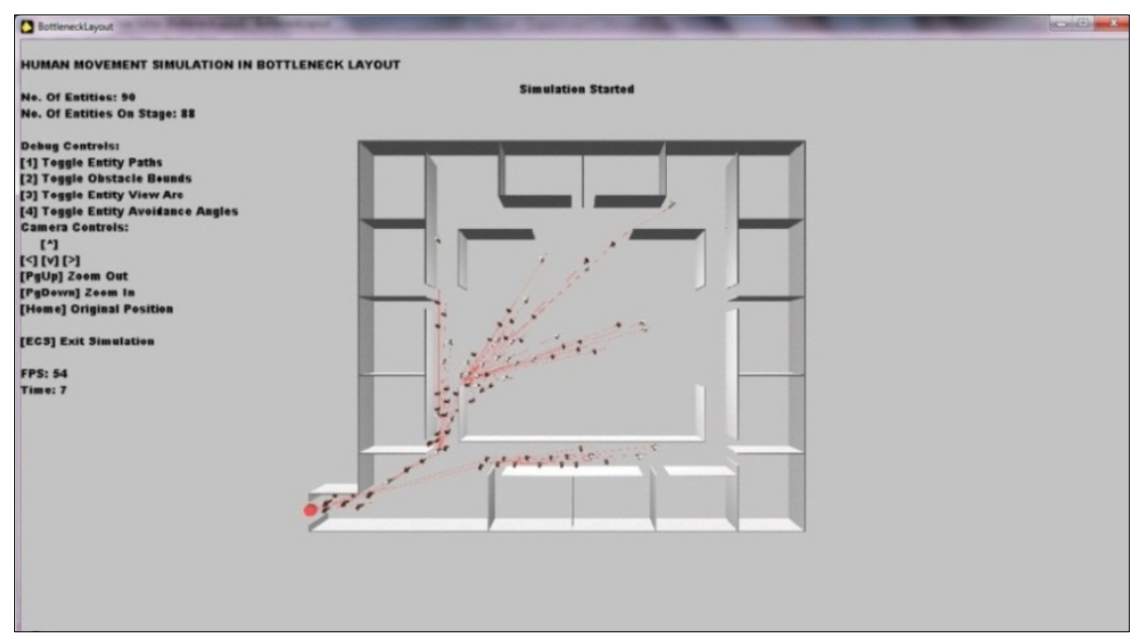

(a) Bottleneck layout

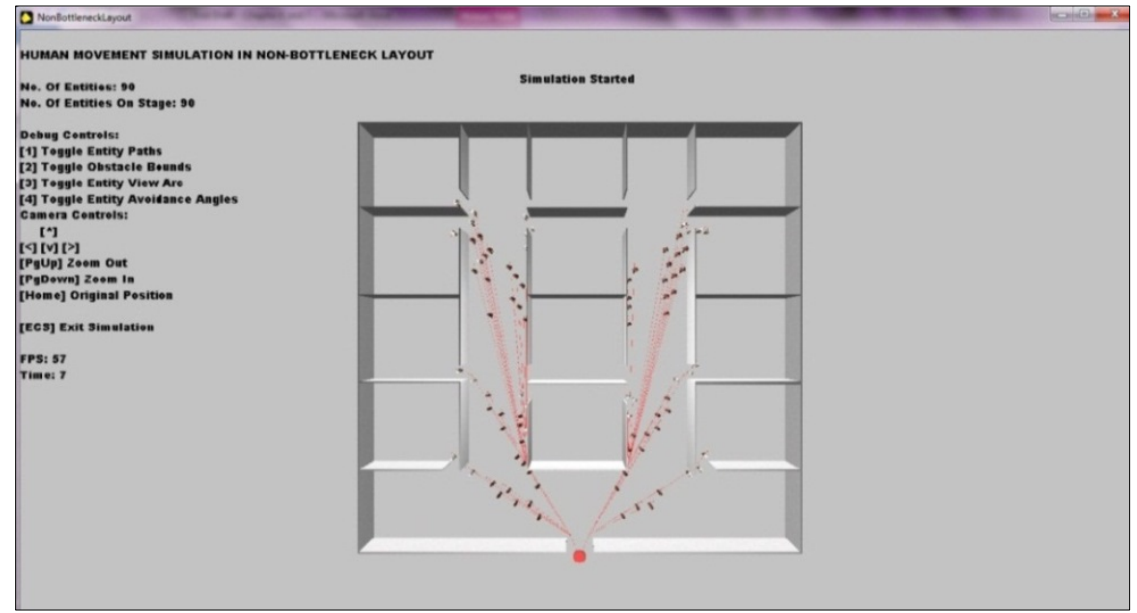

(b) Non-bottleneck layout

Figure 8 Evacuation of entities in bottleneck and non-bottleneck layout

\section{Simulation Results and Discussions}

For Layout 1, the first and last entities arriving at the movement objective moved with an average speed of $0.97 \mathrm{~m} / \mathrm{s}$ and $0.53 \mathrm{~m} / \mathrm{s}$ respectively. For Layout 2, the first and last entities arrived at the movement objective with average speeds of $0.86 \mathrm{~m} / \mathrm{s}$ and $0.60 \mathrm{~m} / \mathrm{s}$ respectively. The results in Figure 9 show that the entities move slightly faster in Layout 1 compared to Layout 2. 


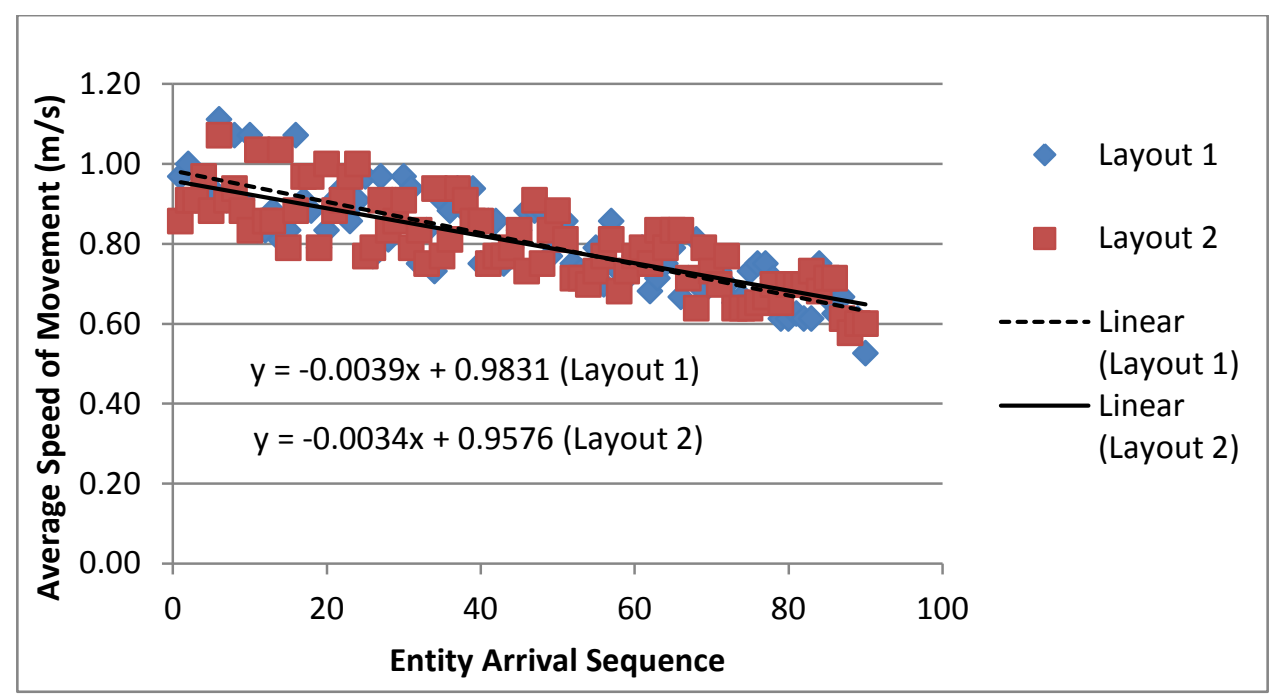

Figure 9 Comparison between Layout 1 and Layout 2 for a multi-mode transportation system simulation

The results indicate that the movement of entities in Layout 2 resulted in more avoidance behaviour compared to the movement of entities in Layout 1. Although a bottleneck area appeared in Layout 1 (the area between the stalls), the bottleneck area was observed to support the rearrangement of the entities' movement. Therefore, the entities were observed to follow more structured movement after avoiding each other compared to Layout 2. In Layout 2, more time was available for the entities to avoid each other.

The results show that the simulation is useful in visualizing different possible layouts of facilities or buildings before real construction or modification is undertaken. The simulation can save time and cost in examining new ideas for improvement or innovation in layout design. The simulation software is also able to predict the effect of different layouts on human movement and behaviour in the crowd. The factors affecting human movement and behaviour obtained from the simulation are useful as an input for crowd research. The simulation also can be used to confirm the crowd observation conducted from the real world.

For bottleneck and non-bottleneck layout evacuation, the simulation results (Figure 10) show that the total evacuation time with the bottleneck layout is higher compared to total evacuation time for the non-bottleneck layout. Although the fastest entities evacuate from both layouts in 10 seconds, entities in the bottleneck layout needed a total of 51 seconds to evacuate from the area while only 36 seconds was needed to evacuate the 
non-bottleneck layout. Additionally, the linear regression line indicates that entities evacuate in a more structured manner from the non-bottleneck layout when compared to evacuation from the bottleneck layout.

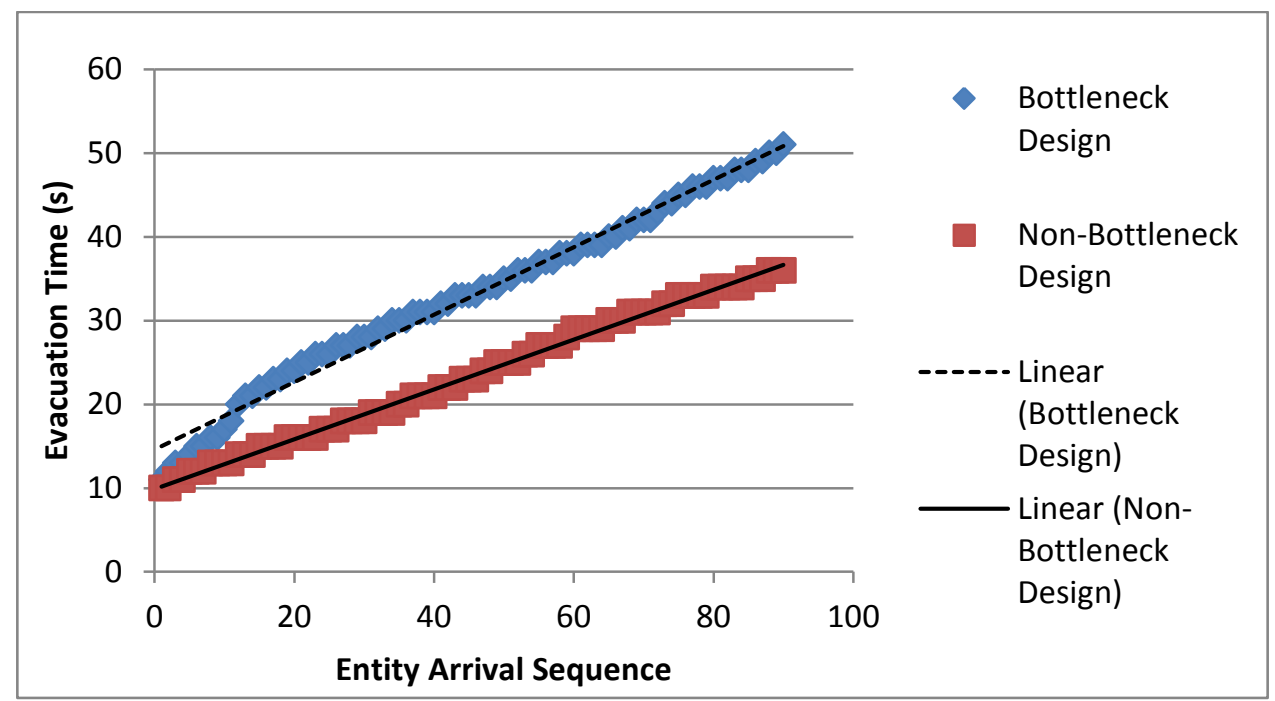

Figure 10 Evacuation time from bottleneck and non-bottleneck layout

The results show that the simulation is useful in providing information on crowd evacuation for crowd safety. The simulation conducted for example indicates that a non-bottleneck layout provides a safer environment in terms of human movement in crowded areas. The simulation is also useful to predict how humans evacuate if there is only one exit door available for the evacuation process. The simulation can also be enhanced in the future by providing other obstacles in the design such as furniture (tables, chairs, etc.) to examine the effect of interior design or arrangement on human movement and behaviour during evacuation.

\section{Conclusions}

An extensive activity of video observation was conducted with nearly 19,000 individual human movements and behaviours being analysed using six hours of video recording. The analysis of the data indicated that human movement can be divided into three major patterns known as free, opposite and same direction. From observation of the three patterns of movement it was possible to derive six different types of behaviour known as Moving Through, Move-Stop-Move, Queuing, Competitive, Avoiding and Passing Through, Queuing and Competitive. 
Six factors were identified as affecting human movement and behaviour based on the video observational analysis and these were Personal Objective, Visual Perception, Speed of Movement, Personal Space, Crowd Density and Avoiding Angle or Distance. The factors can be considered as being interrelated with each other.

Human movement and behaviour in crowded areas is difficult to understand or identify by only analysing recorded video material using simple observation. In this research, Conceptual Behaviours were developed to assist in the process of capturing and analysing human movement and behaviour from the recorded video. At the same time as Conceptual Behaviours formed the basis of the analysis, observational support software was used and an observational database was developed to support the analysis process. Observational support software offers a method to analyse the recorded video frame-by-frame and in slow motion with the ability to play back the video. The observational database keeps the analysis data in a structured form for further reference and analysis. The use of the framework is considered tedious as it takes a long time to analyse the recorded video. However, it offered advantages in understanding 'real' human movement and behaviour and provided considerable insight for this research and any future research.

The simplified human movement and behaviour model (in the real world) was applied to develop the software design model. The software design model is a process to represent or convert human movement and behaviour from the real world to the virtual environment. The development process was modified from the observation and analysis process (modified from the analysis sequence in the real world). Instead of focusing on the movement, behaviour and factors, human movement and behaviour in the virtual environment were modelled based on the sequence of factors, movements and behaviours with selected case studies. The simulation of human movement and behaviour was based on the Agentbased modelling (ABM) approach. ABM uses an object-oriented method where different types of characteristic of (heterogeneous) entities in virtual environment can be modelled. Besides the ability to simulate heterogeneous entities, $\mathrm{ABM}$ can be applied in developing simulation at the microscopic scale where detailed analysis of human movement and behaviour can be considered and measured. ABM is also capable of simulating both normal and emergency situations. DarkBASIC Professional gaming software was selected to simulate human movement and behaviour in the virtual environment as it had artificial intelligence commands to support the movement of entities in the virtual crowd. 
The effectiveness and usefulness of the simulation software was observed through the simulation of the building design consisting of multimode transportation system, bottleneck and non-bottleneck layouts. The simulation software was considered to be beneficial in visualizing different possible layouts in the virtual environment before real construction or modification is conducted in the real world. The method is considered efficient in saving time and cost while providing solutions to produce more idea innovation for building design development. The simulation is also potentially useful for the crowd safety applications such as traffic control and prediction of emergency situations.

\section{References}

Augustijn-Beckers, E.W., Flacke, J. and Retsios, B. (2010). Investigating the Effect of Different Pre-Evacuation Behavior and Exit Choice Strategies Using Agent-based Modeling. Procedia Engineering, Vol. 3, pp.23-35.

Daamen, W., Bovy, P.H.L. and Hoogendoorn, S.P. (2002). Modelling Pedestrians in Transfer Stations. Pedestrian and Evacuation Dynamics, pp.59-73.

Daoling, Z., Lizhong, Y. and Jian, L. (2006). Exit Dynamics of Occupant Evacuation in an Emergency. Physica A: Statistical Mechanics and its Applications, Vol. 363, No. 2, pp.501-511.

David, G., Woods, V., Li, G. and Buckle, P. (2008). The Development of the Quick Exposure Check (QEC) for Assessing Exposure to Risk Factors for Work-related Musculoskeletal Disorders. Applied Ergonomics, Vol. 39, No. 1, pp.57-69

Dempsey, P.G., Mcgorry, R.W. and Maynard, W.S. (2005). A Survey of Tools and Methods used by Certified Professional Ergonomists. Applied Ergonomics, Vol. 36, No. 4, pp.489-503.

Fang, Z., Lo, S. and Lu, J. (2003). On the relationship between crowd density and movement velocity. Fire Safety Journal, Vol. 38, no. 3, pp.271-283.

Friedman, R. (1992). An International Survey of Computer Models for Fire and Smoke. Journal of Fire Protection Engineering, vol. 4, No. 3, pp.81-92. 
Fulton Suri, J. and Marsh, M. (2000). Scenario Building as An Ergonomics Method in Consumer Product Design. Applied Ergonomics, Vol. 31, No. 2, pp.151-157

Helbing, D., Isobe, M., Nagatani, T. and Takimoto, K. (2003). Lattice Gas Simulation of Experimentally Studied Evacuation Dynamics. Physical Review E Vol. 67, No. 6, 067101.

Helbing, D. and Molnar, P. (1995). Social Force Model for Pedestrian Dynamics. Physical Review E, Vol. 51, No. 5, pp.4282-4286

Henein, C.M. and White, T. (2007). Macroscopic Effects of Microscopic Forces Between Agents in Crowd Models. Physica A: Statistical and Theoretical Physics, Vol. 373, pp.694-712.

Hignett, S. and Mcatamney, L. (2000). Rapid Entire Body Assessment (REBA). Applied Ergonomics, Vol. 31, No. 2, pp.201-205.

Mcatamney, L. and Nigel Corlett, E. (1993). RULA: A Survey Method for the Investigation of Work-Related Upper Limb Disorders. Applied Ergonomics, Vol. 24, No. 2, pp.91-99.

Marshall, R., Porter, J. M., Sims, R. E., Summerskill, S. J., Gyi, D. E. and Case, K. (2009). The HADRIAN approach to accessible transport. Work - A Journal of Prevention, Assessment \& Rehabilitation, Vol. 33, No. 3, pp.335-344.

Mehran, R., Oyama, A. and Shah. M. (2009). Abnormal Crowd Behavior Detection Using Social Force Model, IEEE Conference on Computer Vision and Pattern Recognition.

Musse, S.R. and Thalmann, D., (1997). A Model of Human Crowd Behavior: Group Inter-relationship and Collision Detection. Computer Animation and Simulation, Proceedings of the Eurographics Workshop on Eurographics '97.

Nagai, R., Fukamachi, M. and Nagatani, T. (2005). Experiment and Simulation for Counterflow of People Going On All Fours. Physica A: Statistical Mechanics and its Applications, Vol. 358, No. 2, pp.516528.

Noldus, L.P., Trienes, R.J., Hendriksen, A.H., Jansen, H. and Jansen, R.G. (2000). The Observer Video-Pro: New Software for the Collection, Management, and Presentation of Time-Structured Data from Videotapes and Digital Media Files. Behavior Research Methods, Instruments and Computers, Vol. 32, No. 1, pp.197-206. 
Olenick, S.M. and Carpenter, D.J. (2003). An Updated International Survey of Computer Models for Fire and Smoke. Journal of Fire Protection Engineering, Vol. 13, No. 2, pp.87-110.

Ondrej, J., Pettre, J., Olivier, A. and Donikian, S. (2010). A SyntheticVision based Steering Approach for Crowd Simulation. ACM Transactions on Graphics (TOG), Vol. 29, No. 4, pp.123.

Mohamaddan, S. and Case, K. (2012). Towards understanding of human behaviour in crowded spaces. In T. S. Baines, B. T. Clegg and D. K. Harrison (Eds.), 'Advances in Manufacturing Technology XXVI', the Proceedings of the Tenth International Conference on Manufacturing Research, ICMR 2012 Vol. 2, pp.637-642. Aston University, Birmingham, UK, September 11-13, 2012.

Pan, X., Han, C.S., Dauber, K. and Law, K.H. (2006). Human and Social Behavior in Computational Modeling and Analysis of Egress. Automation in Construction, Vol. 15, No. 4, pp.448-461.

Papadimitriou, E., Yannis, G. and Golias, J. (2009). A Critical Assessment of Pedestrian Behaviour Models. Transportation Research Part F: Traffic Psychology and Behaviour, Vol. 12, No. 3, pp.242-255.

Pascual, S.A. and Naqvi, S. (2008). An Investigation of Ergonomics Analysis Tools Used in Industry in the Identification of Work-Related Musculoskeletal Disorders. International Journal of Occupational Safety and Ergonomics, Vol. 14, No. 2, pp.237-245.

Pehkonen, I., Ketola, R., Ranta, R. and Takala, E.P. (2009). A VideoBased Observation Method to Assess Musculoskeletal Load in Kitchen Work. International Journal of Occupational Safety and Ergonomics, Vol. 15, No. 1, pp.75-88

Perez, G.J., Tapang, G., Lim, M. and Saloma, C. (2002). Streaming, Disruptive Interference and Power-Law Behavior in the Exit Dynamics of Confined Pedestrians. Physica A: Statistical Mechanics and its Applications, Vol. 312, No. 3, pp.609-618.

Schadschneider, A. (2002). Cellular Automaton Approach to Pedestrian Dynamics - Theory. Pedestrian and Evacuation Dynamics, pp.75-86.

Schelhorn, T., O’Sullivan, D., Haklay, M. and Thurstain-Goodwin, M. (1999). STREETS: an agent-based pedestrian model.

Schieritz, N. and Milling, P.M. (2003). Modeling the Forest or Modeling the Trees. Proceedings of the 21st International Conference of the System Dynamics Society. 
Seyfried, A., Steffen, B. and Lippert, T. (2006). Basics of Modelling the Pedestrian Flow. Physica A: Statistical Mechanics and its Applications, Vol. 368, No. 1, pp.232-238.

Shirish, S. (2001). Visual Perception - Distinct Human Eye. Available from: http://www.artinarch.com/vp05.html

Still, G.K. (2000). Crowd Dynamics. Doctoral Thesis. University of Warwick.

Syed Shazali, S.T. (2010). Human Behaviour Modelling through Human Intelligent Movement Software (HIMs). Doctoral Thesis. Loughborough University.

Tajima, Y. and Nagatani, T. (2002). Clogging Transition of Pedestrian Flow in T-Shaped Channel. Physica A: Statistical Mechanics and its Applications, Vol. 303, No. 1, pp.239-250.

Tajima, Y., Takimoto, K. and Nagatani, T. (2001). Scaling of Pedestrian Channel Flow with a Bottleneck. Physica A: Statistical Mechanics and its Applications, Vol. 294, No. 1, pp.257-268.

Teknomo, K. (2002). Microscopic Pedestrian Flow Characteristics: Development of An Image Processing Data Collection and Simulation Model. Doctoral Thesis. Tohoku University.

Wai Loon, C. (2011). Motion Detection and Simulation Study on Human Walking Behaviour. Master Thesis, Universiti Malaysia Sarawak.

Zhang, J., Song, W. and Xu, X. (2008). Experiment and Multi-Grid Modeling of Evacuation from a Classroom. Physica A: Statistical Mechanics and its Applications, Vol. 387, No. 23, pp.5901-5909.

Zheng, X., Zhong, T. and Liu, M. (2009). Modeling Crowd Evacuation of a Building Based on Seven Methodological Approaches. Building and Environment, Vol. 44, No. 3, pp.437-445. 


\section{Authors}

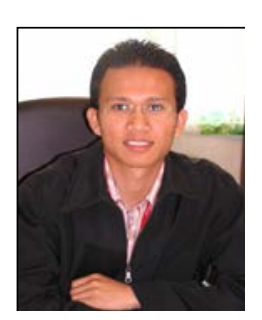

\section{Shahrol Mohamaddan}

Dr Shahrol Mohamaddan is a Senior Lecturer at Department of Mechanical and Manufacturing, Faculty of Engineering, Universiti Malaysia Sarawak (UNIMAS), Malaysia. He completed his Bachelor and Master Degree from Shibaura Institute of Technology, Japan and PhD from Loughborough University, UK. His research interest is in Ergonomics, Robotics and Product Design.

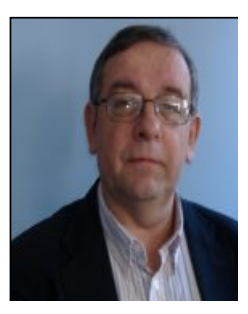

\section{Keith Case}

Professor Keith Case is a Professor of Computer Aided Engineering from Loughborough University, United Kingdom. His research interest is in Computer Aided Design, CAD/CAM Integration, Ergonomics and Inclusive Design. He received the Otto Edholm Award, one of the Ergonomics Society's most prestige awards for his contribution to Ergonomics research. 